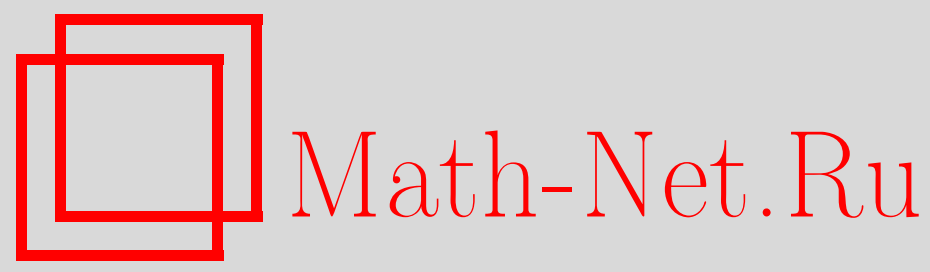

Г. А. Омельянов, В. В. Трушков, Динамика свободной границы в бинарной среде с переменными коэффициентами теплопроводности, Матем. заметки, 1999, том 66, выпуск 2, 231-241

DOI: https://doi.org/10.4213/mzm1161

Использование Общероссийского математического портала Math-Net.Ru подразумевает, что вы прочитали и согласны с пользовательским соглашением http://www . mathnet.ru/rus/agreement

Параметры загрузки:

IP : 54.84 .234 .179

26 апреля 2023 г., 12:50:26

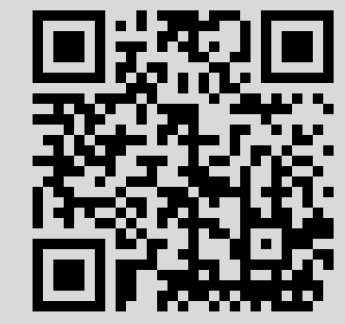




\section{ДИНАМИКА СВОБОДНОЙ ГРАНИЦЫ В БИНАРНОЙ СРЕДЕ С ПЕРЕМЕННЫМИ КОЭФФИЦИЕНТАМИ ТЕПЛОПРОВОДНОСТИ}

\section{Г. А. Омельянов, В. В. Трушков}

В работе построено асимптотическое решение системы уравнений фазового поля с переменными коэффициентами теплопроводности, различными в областях, занятых разными фазами. Показано, что в зависимости от соотношений между параметрами, характеризующими вещество, динамика свободной границы раздела фаз определяется решением классической или модифицированной задачи Стефана.

Библиография: 18 названий.

1. Введение. Система уравнений фазового поля

$$
\begin{gathered}
\frac{\partial \theta}{\partial t}+\frac{l}{2} \frac{\partial \varphi}{\partial t}=K \Delta \theta, \quad x \in \Omega, \quad t>0, \\
\tau \frac{\partial \varphi}{\partial t}=\xi^{2} \Delta \varphi+\frac{1}{a}\left(\varphi-\varphi^{3}\right)+\varkappa(\varphi) \theta
\end{gathered}
$$

впервые была предложена Г. Каджинелпом [1], [2] для упрощенного описания процессов,

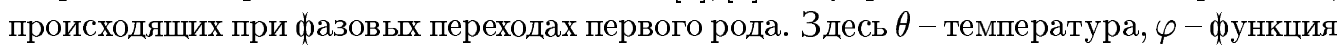
порядка, нормированная так, что двум возможным агрегатным состояниям вещества (назовем их жидкой и твердой фазами) соответствуют значения $\varphi= \pm 1$, параметры $\tau, l, \xi, a, \varkappa, K>0$ характеризуют вещество, $\varkappa(\varphi)$ - некоторая гладкая функция, $\Omega$ - ограниченная область в $\mathbb{R}^{n}$ с гладкой $\left(C^{\infty}\right)$ границей $\partial \Omega$.

Известно, что эта система сохраняет апшроксимационные свойства в различных физических ситуациях, т.е. при различных значениях коэффициентов $l, K, \tau, \xi, a, \varkappa$. В реальных средах величины $a, \xi, \tau$ являются малыми. Трактуя их как малые параметры и переходя к пределу, из исходной модели можно получить предельные задачи, разные при различных соотношениях между $a, \xi$ и $\tau$. В частности, если

$$
a, \xi, \tau \rightarrow 0, \quad a^{-1 / 2} \xi \rightarrow 0, \quad a^{-1} \tau \rightarrow 0,
$$

то, обозначая через $\varepsilon=a$ мальй параметр и полагая $l=2, K=1, \xi=\varepsilon^{5 / 2}, \tau=\varkappa_{1} \varepsilon^{5}$, в качестве предельной при $\varepsilon \rightarrow 0$ задачи получаем классическую задачу Стефана

$$
\begin{gathered}
\frac{\partial \theta^{ \pm}}{\partial t}=\Delta \theta^{ \pm}, \quad t>0, \quad x \in \Omega_{t}^{ \pm}, \\
{\left.\left[\theta^{ \pm}\right]\right|_{\Gamma_{t}}=0,\left.\quad\left[\frac{\partial \theta^{ \pm}}{\partial \nu}\right]\right|_{\Gamma_{t}}=2 V_{\nu},} \\
\left.\theta^{ \pm}\right|_{\Gamma_{t}}=0
\end{gathered}
$$

Работа выполнена при частичной поддержке Российского фонда фундаментальных исследований, грант № 96-01-01492. 
для температур $\theta^{ \pm}$в областях $\Omega_{t}^{ \pm}$, занятых твердой и жидкой фазами соответственно, а также для поверхности $\Gamma_{t}$ раздела фаз, положение которой заранеенеопределено. Здесь $\Omega_{t}^{ \pm}$- подобласти такие, что при всех $t \geqslant 0$ имеем $\Omega=\Omega_{t}^{+} \cup \Omega_{t}^{-} \cup \Gamma_{t}$, причем граница раздела $\Gamma_{t}$ - гладкая поверхность коразмерности 1 , движущаяся в направлении нормали $\nu$ со скоростью $V_{\nu}$. Через $\left.[f]\right|_{\Gamma_{t}}=\left.f\right|_{\Gamma_{t}+0}-\left.f\right|_{\Gamma_{t}-0}$ здесь и далее обозначен скачок функции $f$ на поверхности $\Gamma_{t}$. Задача $(3),(4)$ дополняется естественньми начальньми и граничными (на $\partial \Omega)$ условиями.

Если же

$$
a, \xi, \tau \rightarrow 0, \quad \tau a^{-1}=\text { const }, \quad \xi a^{-1 / 2}=\text { const },
$$

то предельньй переход приводит к модифицированной задаче Стефана (ее также называют задачей Стефана с поверхностным натяжением), в которой условие (4) заменено на условие Гиббса-Томсона

$$
\left.\varkappa_{2} \theta^{ \pm}\right|_{\Gamma_{t}}=\mathscr{K}_{t}+\varkappa_{1} V_{\nu}
$$

где $\mathscr{K}_{t}-$ средняя кривизна $\Gamma_{t}, \varkappa_{1}, \varkappa_{2}>0$ - некоторые константы.

Эти и другие соответствия между системой фазового поля и предельными задачами впервые были установлены в работах Каджинелпа (см., например, [1], [2]) с помощью построения формальных асимптотических решений методом сращивания. В последующие годы появилось много работ, посвященных обоснованию предельного перехода, уточнению класса начальных данных, при которых возникает та или иная предельная задача, обоснованию асимптотических разложений и другим аспектам этой задачи (см. [3]-[16] и библиографию в указанных работах). Однако, в этих работах предполагается, что коэффициенты теплопроводности постоянны и одинаковы в обеих фазах. Кроме того, как правило предполагается, что функция $\varkappa$, характеризующая влияние температуры на функцию порядка, является константой (исключение - работы [2], [7]).

Мы рассмотрим физически более адекватньй вариант системы фазового поля. Именно, рассмотрим случай среды с переменными коэффициентами теплопроводности, различными в разных фазах, и функцию $\varkappa$, локализованную в окрестности границы фазового перехода. В данной статье авторами получены предельные задачи для этой системы в случаях (2) и (5).

Предполагая выполнение соотношений (2) или (5), рассмотрим следующий вариант системы фазового поля:

$$
\begin{gathered}
\frac{\partial(\theta+\varphi)}{\partial t}=\langle\nabla, K(\varphi, x, t) \nabla \theta\rangle, \quad t \in(0, T), \quad x \in \Omega, \\
\varkappa_{1} \varepsilon^{\alpha} \frac{\partial \varphi}{\partial t}=\varepsilon^{\alpha} \Delta \varphi+\varphi-\varphi^{3}+\varepsilon \varkappa \theta\left(1-\varphi^{2}\right) .
\end{gathered}
$$

Здесь

$$
K(\varphi, x, t)=\frac{K^{+}+K^{-}}{2}+\frac{K^{+}-K^{-}}{2} \varphi
$$

$K^{ \pm}=K^{ \pm}(x, t) \in C^{\infty}(Q)$ - заданные функции, причем $K^{ \pm} \geqslant$const $>0, \varepsilon-$ малый параметр, $\varkappa>0, \varkappa_{1}>0$ - некоторые константы, $Q=\Omega \times(0, T)$. Система дополнена естественными начальньпи и краевыми условиями

$$
\begin{gathered}
\left.\theta\right|_{t=0}=\theta^{0}(x, \varepsilon),\left.\quad \varphi\right|_{t=0}=\varphi^{0}(x, \varepsilon), \\
\left.\theta\right|_{\Sigma}=\left.h(x, t)\right|_{\Sigma},\left.\quad \varphi\right|_{\Sigma}=1
\end{gathered}
$$


где $h \in C^{\infty}(\Sigma), \Sigma=\partial \Omega \times[0, T]$.

Предполагается, что при $\varepsilon \geqslant$ const $>0$ функции $\theta^{0}$ и $\varphi^{0}$ являются гладкими и заданы подобласти $\Omega_{0}^{ \pm} \subset \Omega$, разделенныегранищей $\Gamma_{0}$, такие, что $\theta^{0}(x, \varepsilon) \rightarrow \theta_{0}^{ \pm}(x)$ и $\varphi^{0}(x, \varepsilon) \rightarrow$ \pm 1 при $x \in \Omega_{0}^{ \pm}$и $\varepsilon \rightarrow 0$. Здесь $\Gamma_{0}$ - замкнутое гладкое многообразие коразмерности 1 , причем $\Omega=\Omega_{0}^{+} \cup \Omega_{0}^{-} \cup \Gamma_{0}$. Кроме того, предположим, что $\theta_{0}^{ \pm} \in C^{\infty}\left(\bar{\Omega}_{0}^{ \pm}\right)$и $\left.\left[\theta_{0}^{ \pm}\right]\right|_{\Gamma_{0}}=0$. Для определенности будем считать, что к внешней границе $\partial \Omega$ примькает $\Omega_{0}^{+}$.

Рассматривая (7)-(10) при $\alpha=6$ и $\alpha=2$ и строя асимптотическое решение, мы получим предельные (при $\varepsilon \rightarrow 0$ ) задачи, которые являются естественньм обобщением классической $(3),(4)$ и модифицированной $(3),(6)$ задач Стефана на случай переменньх коэффициентов теплопроводности.

Построение асимптотики проводится по схеме, развитой в [14], [16]-[18].

2. Классическая модель Стефана. Пусть вьполнено условие (2). Тогда, полагая $\varepsilon=a, \tau=\varkappa_{1} \varepsilon^{5}, \xi=\varepsilon^{5 / 2}$, получаем систему (7), (8) с параметром $\alpha=6$.

Введем вспомогательные функции, необходимые для формулировки основного результата этого пункта. Пусть $\theta^{ \pm}=\theta^{ \pm}(x, \psi), \psi=\psi(x)$ - решение классической задачи Стефана

$$
\begin{gathered}
\frac{\partial \theta^{ \pm}}{\partial t}=\left\langle\nabla, K^{ \pm} \nabla \theta^{ \pm}\right\rangle, \quad t>0, \quad x \in \Omega_{t}^{ \pm}, \\
\left.\theta^{ \pm}\right|_{\Gamma_{t}}=0,\left.\quad\left[K^{ \pm} \frac{\partial \theta^{ \pm}}{\partial \nu}\right]\right|_{\Gamma_{t}}=2 V_{\nu}, \\
\left.\theta^{ \pm}\right|_{t=0}=\theta_{ \pm}^{0},\left.\quad \theta^{+}\right|_{\Sigma}=\left.h(x, t)\right|_{\Sigma} .
\end{gathered}
$$

Здесь предполагается, что нормальная скорость $V_{\nu} \neq 0$, а значит, положение свободной границы $\Gamma_{t}$ можно описать как поверхность уровня вспомогательной функции $\psi(x)$ : $\Gamma_{t}=\{x \in \Omega, \psi(x)=t\}$. Поэтому $V_{\nu}=1 /|\nabla \psi|$ и нормаль к $\Gamma_{t}$ вычисляется по формуле $\nu=\nabla \psi /|\nabla \psi|$. Будем предполагать, что при $t \leqslant T$ сушествует классическое решение задачи (11)-(13).

Нам также потребуются функции $\widetilde{\theta}^{ \pm}$- гладкие продолжения $\theta^{ \pm}$в области $\Omega_{t}^{\mp}$ соответственно такие, что $\widetilde{\theta}^{ \pm}(x, t)=\theta^{ \pm}(x, t)$ при $x \in \bar{\Omega}_{t}^{ \pm}$и $\widetilde{\theta}^{ \pm}$удовлетворяют уравнению теплопроводности (11) в $\varepsilon^{3}$-окрестности свободной гранищы $\Gamma_{t}$. Вне этой окрестности выбор продолжения несущественен.

Далее, пусть $\theta_{1}^{ \pm}=\theta_{1}^{ \pm}(x, \psi), \psi_{1}=\psi_{1}(x)$ - решение линеаризованной задачи Стефана

$$
\begin{gathered}
\frac{\partial \theta_{1}^{ \pm}}{\partial t}=\left\langle\nabla, K^{ \pm} \nabla \theta_{1}^{ \pm}\right\rangle, \quad t>0, \quad x \in \Omega_{t}^{ \pm}, \\
{\left.\left[\theta_{1}^{ \pm}\right]\right|_{\Gamma_{t}}=0,\left.\quad \theta_{1}^{ \pm}\right|_{t=0}=\theta_{ \pm}^{1},\left.\quad \theta_{1}^{+}\right|_{\Sigma}=0,} \\
\theta_{1}^{ \pm}-\left.\psi_{1} \frac{1}{2}\left(\frac{\partial \theta^{+}}{\partial t}+\frac{\partial \theta^{-}}{\partial t}\right)\right|_{\Gamma_{t}}=0,\left.\quad\left[K^{ \pm} \frac{\partial \theta_{1}^{ \pm}}{\partial \nu}\right]\right|_{\Gamma_{t}}=\left.\left[K^{ \pm} \frac{\partial \theta^{ \pm}}{\partial \nu_{1}}\right]\right|_{\Gamma_{t}}+V_{\nu} f,
\end{gathered}
$$

где

$$
\nu_{1}=\frac{\nabla \psi_{1}}{|\nabla \psi|}, \quad \theta_{ \pm}^{1}=\lim _{\varepsilon \rightarrow 0} \frac{\theta^{0}(x, \varepsilon)-\theta_{ \pm}^{0}(x)}{\varepsilon}, \quad x \in \Omega_{0}^{ \pm},
$$

способ вычисления правой части $f$ будет указан ниже. Пусть $\widetilde{\theta}_{1}^{ \pm}(x, t)-$ гладкие продолжения $\theta_{1}^{ \pm}$в области $\Omega_{t}^{\mp}$ соответственно такие, что каждая из функций $\widetilde{\theta}_{1}^{ \pm}$удовлетворяет уравнению теплопроводности (14). 
ТЕОРема 1. Пусть при $t \leqslant T$ существует классическое решение задачи (11)-(13). Тогда при $t \leqslant T$ самоподобное асимптотическое решение типа кинка системы (7), (8) при $\alpha=6$ имеет вид

$$
\begin{aligned}
& \varphi(x, t, \varepsilon)=\chi(\tau, x)+\varepsilon\left\langle\nu, \nu_{1}\right\rangle \tau \frac{\partial \chi(\tau, x)}{\partial \tau}+O\left(\varepsilon^{2}\right) \\
& \theta(x, t, \varepsilon)=\frac{1}{2}\left(\widetilde{\theta}^{+}(x, t)+\widetilde{\theta}^{-}(x, t)\right)+\frac{1}{2}\left(\widetilde{\theta}^{+}(x, t)-\widetilde{\theta}^{-}(x, t)\right) \chi(\tau, x) \\
&+\varepsilon\left(\frac{1}{2}\left(\widetilde{\theta}_{1}^{+}(x, t)+\widetilde{\theta}_{1}^{-}(x, t)\right)+\frac{1}{2}\left(\widetilde{\theta}_{1}^{+}(x, t)-\widetilde{\theta}_{1}^{-}(x, t)\right) \chi(\tau, x)\right. \\
&\left.+\psi_{1}\left(\frac{\partial \widetilde{\theta}^{+}(x, t)}{\partial t}-\frac{\partial \widetilde{\theta}^{-}(x, t)}{\partial t}\right)(\chi(\tau, x)+1)\right)+O\left(\varepsilon^{2}\right)
\end{aligned}
$$

$2 \partial e$

$$
\tau=\frac{t-\psi(x)+\varepsilon \psi_{1}(x)+\varepsilon^{2} \psi_{2}(x, \varepsilon)}{\varepsilon^{3}}, \quad \chi(\tau, x)=\operatorname{th} \frac{\tau}{\sqrt{2}|\nabla \psi|}, \quad \nu_{1}=\frac{\nabla \psi_{1}}{|\nabla \psi|},
$$

$\psi_{2}=\psi_{2}(x, \varepsilon)$ - некоторая гладкая равномерно по $\varepsilon \in[0,1]$ функиия, способ вычисления которой будет указан ниже.

Наметим доказательство теоремы.

Для построения асимптотического решения нам потребуется два класса функций: $\mathscr{S}=\left\{f: f(\tau, x, t) \in S\left(\mathbb{R}^{1} ; C^{\infty}(Q)\right)\right\}$, где $S$ - пространство Шварца, и $\mathscr{H}=\{f:$ $\partial f / \partial \tau \in \mathscr{S}\}$. Ясно, что для любой функции $f \in \mathscr{H}$ существуют пределы

$$
f^{ \pm}(x, t)=\lim _{\tau \rightarrow \pm \infty} f(\tau, x, t), \quad f^{ \pm} \in C^{\infty}(Q)
$$

Нетрудно доказать также следуюшие утверждения (см. [17]).

Лемма 1. 1) Для любых функиий $S(x, t) \in C^{\infty}(Q)$ maких, что

$$
\beta=\left.\frac{\partial S}{\partial t}\right|_{\Gamma_{t}} \neq 0
$$

әде $\Gamma_{t}=\{(x, t) \in Q, S(x, t)=0\}$, июбых функиий $f(\tau, x, t) \in \mathscr{H}$ справедливо соотношение

$$
f\left(\frac{S(x, t)}{\varepsilon}, x, t\right)=f\left(\frac{\beta(t-\psi(x))}{\varepsilon}, x, t\right)+O(\varepsilon),
$$

где $t=\psi(x)$ - уравнение поверхности $S(x, t)=0$.

2) Пусть $\chi(\tau, x, t)$ - некоторая функиия из $\mathscr{H}$ такая, что $\chi^{ \pm}= \pm 1$. Тогда для любой функиии $f \in \mathscr{H}$ справедлива формула

$$
f=\frac{f^{+}+f^{-}}{2}+\frac{f^{+}-f^{-}}{2} \chi(\tau, x, t)+\omega(\tau, x, t)
$$

əде $\omega \in \mathscr{S}$. 
3) Для любых функиий $f(\tau, x, t) \in \mathscr{S}, g(x, t) \in C^{\infty}$ справедливы соотношения

$$
\begin{gathered}
(t-\psi(x))^{k} f\left(\frac{t-\psi(x)}{\varepsilon}, x, t\right)=O\left(\varepsilon^{k}\right), \quad k \geqslant 0, \\
g(x, t) f\left(\frac{t-\psi(x)}{\varepsilon}, x, t\right)=g(x, \psi) f\left(\frac{t-\psi(x)}{\varepsilon}, x, \psi\right)+O(\varepsilon) .
\end{gathered}
$$

Перейдем к построению самоподобного асимптотического решения уравнений (7), (8). Самоподобность в данном случае означает, что решение при $\varepsilon \rightarrow 0$ остается жестко фроновым, т.е. существуют подобласти $\Omega_{t}^{ \pm}$такие, что $\Omega=\Omega_{t}^{+} \cup \Omega_{t}^{-} \cup \Gamma_{t}, \Gamma_{t}=\bar{\Omega}_{t}^{+} \cap \bar{\Omega}_{t}^{-}-$ замкнутое многообразие коразмерности 1 , причем предполагается, что $\Gamma_{t} \cap \partial \Omega=\varnothing$. Будем также считать, что нормальная скорость движения $\Gamma_{t}$ отлична от 0.

Для выбора формы асимптотического решения будем исходить из физического смысла задачи. Ясно, что предел при $\varepsilon \rightarrow 0$ главного члена асимптотики $\varphi$ должен быть разрывным на $\Gamma_{t}$, т.е. иметь особенность типа функции Хевисайда, а предел главного члена асимптотики $\theta$ должен иметь слабую особенность, т.е. быть непрерьвной функцией и в то же время его производная по нормали к $\Gamma_{t}$ должна иметь сильньй разрыв на $\Gamma_{t}$.

Тем самым, мы приходим к следуюшей форме искомого асимптотического решения:

$$
\begin{aligned}
& \theta(x, t, \varepsilon)=\Theta^{N}(x, t, \varepsilon)+\mathscr{V}^{N}\left(\frac{S(x, t, \varepsilon)}{\varepsilon^{3}}, x, t, \varepsilon\right)+O\left(\varepsilon^{N+1}\right), \\
& \varphi(x, t, \varepsilon)=\Phi^{N}\left(\frac{S(x, t, \varepsilon)}{\varepsilon^{3}}, x, t, \varepsilon\right)+O\left(\varepsilon^{N+1}\right),
\end{aligned}
$$

которое можно понимать как регуляризацию описанных вьше предельных функций. Здесь $\Theta^{N}$ - регулярная составляющая решения,

$$
\Theta^{N}(x, t, \varepsilon)=\sum_{j=0}^{N} \varepsilon^{j} \theta_{j}(x, t),
$$

$\mathscr{V}^{N}, \Phi^{N}$ - быстро меняющиеся составляющие решения,

$$
\begin{aligned}
& \mathscr{V}^{N}(\tau, x, t, \varepsilon)=\sum_{j=0}^{N} \varepsilon^{j} f_{j}(x, t) V_{j}(\tau, x, t)+\sum_{j=1}^{N} \varepsilon^{j} g_{j}(\tau, x, t), \\
& \Phi^{N}(\tau, x, t, \varepsilon)=\chi(\tau, x)+\sum_{j=1}^{N} \varepsilon^{j} \varphi_{j}(\tau, x, t),
\end{aligned}
$$

где $\theta_{j}, f_{j}, S \in C^{\infty}(Q), \chi(\tau, x), \varphi_{j}(\tau, x, t), g_{j}(\tau, x, t) \in \mathscr{H}$ - подлежащие определению функции, причем

$$
\chi^{ \pm}= \pm 1,\left.\quad \frac{\partial S}{\partial t}\right|_{\Gamma_{t}} \neq 0, \quad \Gamma_{t}=\{(x, t), t=\psi(x)\},\left.\quad f_{j}\right|_{\Gamma_{t}}=0, \quad g_{j}^{-}=0 .
$$

В силу леммы 1 и (20) без потери общности полагаем $S=t-\psi(x)+\varepsilon \widetilde{\psi}(x, \varepsilon)$, где $\widetilde{\psi}=\psi_{1}(x)+\varepsilon \psi_{2}(x)+\cdots$. 
Подставим разложения (18), (19) в (7), (8). Умножая первое уравнение на $\varepsilon^{6}$, получаем соотношения

$$
\begin{aligned}
& |\nabla S|^{2} \frac{\partial}{\partial \tau}\left(K\left(\Phi^{N}\right) \frac{\partial^{N}}{\partial \tau}\right)+\varepsilon^{3}\left(K\left(\Phi^{N}\right)(2\langle\nabla S, \nabla\rangle+\Delta S)+\left\langle\nabla K\left(\Phi^{N}\right), \nabla S\right\rangle\right. \\
& \left.\quad+\frac{\partial K\left(\Phi^{N}\right)}{\partial \tau}\langle\nabla S, \nabla\rangle\right) \frac{\partial^{N}}{\partial \tau}-\varepsilon^{3} \frac{\partial\left(\mathscr{V}^{N}+\Phi^{N}\right)}{\partial \tau}+\varepsilon^{6}\left\langle\nabla,\left(K\left(\Phi^{N}\right) \nabla\left(\Theta^{N}+\mathscr{V}^{N}\right)\right)\right\rangle \\
& \quad-\left.\varepsilon^{6} \frac{\partial}{\partial t}\left(\Theta^{N}+\mathscr{V}^{N}+\Phi^{N}\right)\right|_{\tau=S / \varepsilon^{3}}=O\left(\varepsilon^{N+7}\right), \\
& \Phi^{N}\left(1-\left(\Phi^{N}\right)^{2}\right)+|\nabla S|^{2} \frac{\partial^{2} \Phi^{N}}{\partial \tau^{2}}+\varepsilon \varkappa\left(\Theta^{N}+\mathscr{V}^{N}\right)\left(1-\left(\Phi^{N}\right)^{2}\right)+\varepsilon^{3}(2\langle\nabla S, \nabla\rangle+\Delta S) \frac{\partial \Phi^{N}}{\partial \tau} \\
& \quad-\varepsilon^{3} \varkappa_{1} \frac{\partial \Phi^{N}}{\partial \tau}+\varepsilon^{6}\left(\Delta-\varkappa_{1} \frac{\partial}{\partial t}\right) \Phi^{N}=O\left(\varepsilon^{N+1}\right) .
\end{aligned}
$$

Определим сначала регулярные члены разложений (18), (19). Переходя к пределу в $(21),(22)$ при $\tau \rightarrow \pm \infty$, получаем соотношения

$$
\begin{gathered}
\left\langle\nabla,\left(K\left(\Phi^{N \pm}\right) \nabla\left(\Theta^{N}+\mathscr{V}^{N \pm}\right)\right)\right\rangle-\frac{\partial}{\partial t}\left(\Theta^{N}+\mathscr{V}^{N \pm}+\Phi^{N \pm}\right)=O\left(\varepsilon^{N+1}\right), \\
\Phi^{N \pm}\left(1-\left(\Phi^{N \pm}\right)^{2}\right)+\varepsilon \varkappa\left(\Theta^{N}+\mathscr{V}^{N \pm}\right)\left(1-\left(\Phi^{N \pm}\right)^{2}\right)+\varepsilon^{6}\left(\Delta-\frac{\partial}{\partial t}\right) \Phi^{N \pm}=O\left(\varepsilon^{N+1}\right) .
\end{gathered}
$$

Приравнивая к 0 коэффициенты при одинаковых степенях $\varepsilon$ в (24), получаем, что $\chi^{ \pm}$ должны быть равны \pm 1 , а также должны вьполняться равенства $\varphi_{j}^{ \pm}=0, j=1, \ldots, N$, т.е. необходимо $\varphi_{j} \in \mathscr{S}$. Кроме того, из (23) мы получаем уравнения

$$
\frac{\partial\left(\theta_{0}+f_{0} V_{0}^{ \pm}\right)}{\partial t}-\left\langle\nabla, K^{ \pm} \nabla\left(\theta_{0}+f_{0} V_{0}^{ \pm}\right)\right\rangle=0
$$

в областях $\Omega_{t}^{ \pm}$соответственно. Обозначая $\theta^{ \pm}=\theta_{0}+f_{0} V_{0}^{ \pm}$, из (25.0) получаем уравнения теплопроводности (11). Рассматривая в (23) члены величины $O\left(\varepsilon^{j}\right), j=1, \ldots, N-1$, обозначая $\theta_{j}^{ \pm}=\theta_{j}+f_{j} V_{j}^{ \pm}+g_{j}^{ \pm}$и принимая во внимание, что $\varphi_{j}^{ \pm}=0$, получаем уравнения

$$
\frac{\partial \theta_{j}^{ \pm}}{\partial t}-\left\langle\nabla, K^{ \pm} \nabla \theta_{j}^{ \pm}\right\rangle=0, \quad j=1, \ldots, N-1 .
$$

Построение быстро меняющихся составляющих решения $\mathcal{V}^{N}, \Phi^{N}$ проведем по схеме, развитой в [14], [17]: последовательно для всех членов $(21),(22)$ величины $O\left(\varepsilon^{j}\right)$, $j=1, \ldots, N$, раскладываем в ряд Тейлора по $t$ в точке $t=\psi(x)$ и используем равенство $\tau=(t-\psi(x)+\varepsilon \widetilde{\psi}(x)) / \varepsilon^{3}$. Затем переходим к функциям с независимыми переменными $\tau, x$ и находим решение на поверхности $\Gamma_{t}$, т.е. при $t=\psi(x)$. Далее строим продолжение функций вне $\Gamma_{t}$ так, чтобы обеспечить существование младших членов асимптотики, обладающих требуемыми свойствами. Ввиду того что $\varphi_{j} \in \mathscr{S}$, определим продолжение $\varphi_{j}$ как тождественное, т.е. $\varphi_{j}=\varphi_{j}(\tau, x)$.

Реализуя эту схему, рассмотрим члены величины $O(1)$ в соотношении (22). Приравнивая их к 0 , получаем модельное уравнение для $\check{\chi}=\chi(\tau, x, \psi(x))$ :

$$
|\nabla \psi|^{2} \frac{\partial^{2} \check{\chi}}{\partial \tau^{2}}+\check{\chi}-\check{\chi}^{3}=0, \quad \check{\chi} \rightarrow 1 \text { при } \tau \rightarrow+\infty .
$$


Отсюда на $\Gamma_{t}$ имеем

$$
\check{\chi}(\tau, x)=\operatorname{th} \frac{\tau}{\sqrt{2}|\nabla \psi|},
$$

причем возникающую при интегрировании (26) "постоянную" интегрирования $c=$ $c(x)$ мы включаем во входящую в разложение $S$ и пока не определенную функцию $\psi_{3}(x)$. Определим продолжение $\check{\chi}$ для всех $(x, t) \in Q$ как тождественное, т.е. положим $\chi=\check{\chi}(\tau, x)$, поскольку именно такое продолжение согласуется с полученным выше необходимым условием $\chi^{ \pm}= \pm 1$.

Далее, приравнивая члены величины $O\left(\varepsilon^{1}\right)$ в соотношениях $(22)$ и $(21)$, получаем уравнения

$$
\begin{gathered}
\widehat{\mathscr{L}} \varphi_{k}=F_{k}^{\varphi}(\tau, x) \\
|\nabla \psi|^{2} \frac{\partial}{\partial \tau}\left(K(\chi) \frac{\partial \check{g}_{k}}{\partial \tau}\right)=F_{k}^{\theta}(\tau, x) .
\end{gathered}
$$

Здесь

$$
\widehat{\mathscr{L}}=|\nabla \psi|^{2} \frac{\partial^{2}}{\partial \tau^{2}}+1-3 \chi^{2}
$$

$F_{k}^{\varphi}, F_{k}^{\theta}$ - функции от $x, \tau, \chi, \ldots, \varphi_{k-1}, g_{k-1}$ и их производных. В частности,

$$
\begin{aligned}
F_{1}^{\varphi}(\tau, x)= & 2\left\langle\nabla \psi, \nabla \psi_{1}\right\rangle \frac{\partial^{2} \chi}{\partial \tau^{2}}-\left.\varkappa \theta_{0}\left(1-\chi^{2}\right)\right|_{\Gamma_{t}}, \\
F_{1}^{\theta}= & \left.|\nabla \psi|^{2} \frac{\partial}{\partial \tau} K(\chi) \psi_{1} \frac{\partial f_{0}}{\partial t} \frac{\partial V_{0}}{\partial \tau}\right|_{\Gamma_{t}}, \\
F_{2}^{\theta}= & \left.|\nabla \psi|^{2} \frac{\partial}{\partial \tau} K(\chi)\left(\psi_{1} \frac{\partial f_{1}}{\partial t} \frac{\partial V_{1}}{\partial \tau}+\psi_{2} \frac{\partial f_{0}}{\partial t} \frac{\partial V_{0}}{\partial \tau}-\psi_{1}^{2} \frac{1}{2} \frac{\partial^{2} f_{0}}{\partial t^{2}} \frac{\partial V_{0}}{\partial \tau}\right)\right|_{\Gamma_{t}}, \\
F_{3}^{\theta}(\tau, x)= & |\nabla \psi|^{2} \frac{\partial}{\partial \tau} K(\chi)\left(\psi_{1} \frac{\partial f_{2}}{\partial t} \frac{\partial V_{2}}{\partial \tau}+\psi_{2} \frac{\partial f_{1}}{\partial t} \frac{\partial V_{1}}{\partial \tau}+\psi_{3} \frac{\partial f_{0}}{\partial t} \frac{\partial V_{0}}{\partial \tau}\right. \\
& \left.-\psi_{1}^{2} \frac{1}{2} \frac{\partial^{2} f_{1}}{\partial t^{2}} \frac{\partial V_{1}}{\partial \tau}-\psi_{1} \psi_{2} \frac{1}{2} \frac{\partial^{2} f_{0}}{\partial t^{2}} \frac{\partial V_{0}}{\partial \tau}+\psi_{1}^{3} \frac{1}{6} \frac{\partial^{3} f_{0}}{\partial t^{3}} \frac{\partial V_{0}}{\partial \tau}\right)\left.\right|_{\Gamma_{t}} \\
& +\left.\frac{\partial}{\partial \tau}\left(\chi+K(\chi)\left\langle\nabla \psi, \nabla\left(\theta_{0}+f_{0} V_{0}\right)\right\rangle\right)\right|_{\Gamma_{t}} \cdot
\end{aligned}
$$

Нетрудно установить справедливость следующего утверждения.

Лемма 2. Для разрешимости уравнений $(27 . k),(28 . k)$ в $\mathscr{H}$ необходимо и достаточно выполнение условий

$$
\begin{gathered}
F_{k}^{\theta} \in \mathscr{S}, \\
F_{k}^{\varphi} \in \mathscr{S} \\
\int_{-\infty}^{+\infty} F_{k}^{\theta}(\tau, x) d \tau=0, \\
\int_{-\infty}^{+\infty} F_{k}^{\varphi}(\tau, x) \frac{\partial \chi}{\partial \tau} d \tau=0 .
\end{gathered}
$$


В силу (29.1) условие (32.1), очевидно, вьполнено, а условие (34.1) вьполнено тогда и только тогда, когда $\left.\theta_{0}\right|_{\Gamma_{t}}=0$. Так как $\check{f}_{0}=0$, из этого равенства получаем условие Стефана (12).

Теперь заметим, что в качестве $V_{j}$ можно выбрать функцию $\chi$, поскольку в силу леммы 1 разность между двумя кинками с одинаковьми пределами на $\pm \infty$ является функцией из $\mathscr{S}$, а значит, учитьвается в слагаемом $g_{j}$ разложения $(18)$.

Определим продолжение функции $\left.\left(\theta_{0}+f_{0} V_{0}\right)\right|_{\Gamma_{t}}$ вне поверхности $\Gamma_{t}$. Для этого пусть $\theta^{ \pm}=\theta^{ \pm}(x, t)-$ решение задачи Стефана в $\Omega_{t}^{ \pm}$и $\widetilde{\theta}^{ \pm}=\widetilde{\theta}^{ \pm}(x, t)$ - гладкие продолжения $\theta^{ \pm}$в области $\Omega_{t}^{\mp}$ соответственно такие, что $\widetilde{\theta}^{ \pm}$также удовлетворяют уравнению теплопроводности (25.0). Положим $f_{0} V_{0}=\left(\widetilde{\theta}^{+}-\widetilde{\theta}^{-}\right) \chi / 2, \theta_{0}=\left(\widetilde{\theta}^{+}+\widetilde{\theta}^{-}\right) / 2$ и определим в $\Omega$ главный член асимптотики $\theta$ :

$$
\theta(x, t, \varepsilon)=\frac{\tilde{\theta}^{+}+\tilde{\theta}^{-}}{2}+\frac{\left(\tilde{\theta}^{+}-\tilde{\theta}^{-}\right) \chi(\tau, x)}{2}+O(\varepsilon) .
$$

Решая уравнение (27.1), получаем

$$
\varphi_{1}=\frac{\left\langle\nabla \psi, \nabla \psi_{1}\right\rangle}{|\nabla \psi|^{2}} \tau \frac{\partial \chi}{\partial \tau}
$$

Здесь мы вновь используем тот факт, что фаза $S$ содержит члены вида $\varepsilon^{j} \psi_{j}$, в частности, член $\varepsilon^{4} \psi_{4}$. Ясно, что учет этого слагаемого в $S$ эквивалентен добавлению к $(35)$ функции $c_{1}(x) \chi_{\tau} \in \operatorname{ker} \widehat{\mathscr{L}}$.

Теперь рассмотрим уравнения $(28.1),(28.2),(28.3)$. В силу явного вида функций $F_{i}^{\theta}$, условия $(31 . i),(33.1)$ и (33.2) также выполнены. Уравнения $(28.1),(28.2)$ легко решаются

$$
\begin{aligned}
& \check{g}_{1}=\left.\psi_{1} \frac{\partial f_{0}}{\partial t}\right|_{\Gamma_{t}}(\chi+1), \\
& \check{g}_{2}=\left.\left(\psi_{1} \frac{\partial f_{1}}{\partial t}+\psi_{2} \frac{\partial f_{0}}{\partial t}-\psi_{1}^{2} \frac{1}{2} \frac{\partial^{2} f_{0}}{\partial t^{2}}\right)\right|_{\Gamma_{t}}(\chi+1) .
\end{aligned}
$$

Далее, проинтегрируем (28.3) по $\tau$ :

$$
\begin{aligned}
& |\nabla \psi|^{2} K(\chi)\left(\frac { \partial } { \partial \tau } \left(\check{g}_{3}-\left(\psi_{1} \frac{\partial f_{2}}{\partial t}+\psi_{2} \frac{\partial f_{1}}{\partial t}+\psi_{3} \frac{\partial f_{0}}{\partial t}-\psi_{1}^{2} \frac{1}{2} \frac{\partial f_{1}}{\partial t}\right.\right.\right. \\
& \left.\left.\left.\quad-\psi_{1} \psi_{2} \frac{\partial f_{0}}{\partial t}+\psi_{1}^{3} \frac{1}{6} \frac{\partial^{3} f_{1}}{\partial t^{3}}\right) \chi\right)-\left\langle\nabla \psi, \nabla\left(\theta_{0}+f_{0} V_{0}\right)\right\rangle\right)\left.\right|_{\Gamma_{t}}=\chi+c^{(1)}
\end{aligned}
$$

где $c^{(1)}=c^{(1)}(x)$ - "постоянная" интегрирования. Для того чтобы удовлетворить условие (33.3), устремим в (36) $\tau$ к $\pm \infty$ и, используя тривиальные арифметические операции, получим условие

$$
\left.\left(K^{+}\left\langle\nabla \psi, \nabla\left(\theta_{0}+f_{0} V_{0}^{+}\right)\right\rangle-K^{-}\left\langle\nabla \psi, \nabla\left(\theta_{0}+f_{0} V_{0}^{-}\right)\right\rangle\right)\right|_{\Gamma_{t}}=\frac{2}{|\nabla \psi|} .
$$

Нетрудно убедиться, что условие (37) эквивалентно условию Стефана (12) для производной по нормали. 
Далее, приравнивая в (22) члены величины $O\left(\varepsilon^{2}\right)$, получаем $(27.2)$ с правой частью

$$
\begin{aligned}
F_{2}^{\varphi}= & \left(3 \chi \varphi_{1}^{2}-\theta_{1}\left(1-\chi^{2}\right)+\varkappa \frac{\partial\left(\theta_{0}+f_{0} V_{0}\right)}{\partial t} \psi_{1}\left(1-\chi^{2}\right)\right. \\
& \left.-\varkappa \theta_{1}\left(1-\chi^{2}\right)+2\left\langle\nabla \psi, \nabla \psi_{1}\right\rangle \frac{\partial^{2} \varphi_{1}}{\partial \tau^{2}}-\left(\left|\nabla \psi_{1}\right|^{2}-2\left\langle\nabla \psi, \nabla \psi_{2}\right\rangle\right) \frac{\partial^{2} \chi}{\partial \tau^{2}}\right)\left.\right|_{\Gamma_{t}} .
\end{aligned}
$$

С учетом (35) нетрудно убедиться, что условие (34.2) вьполнено тогда и только тогда, когда

$$
\check{\theta}_{1}-\left.\psi_{1} \frac{1}{2}\left(\frac{\partial \widetilde{\theta}^{+}}{\partial t}+\frac{\partial \widetilde{\theta}^{-}}{\partial t}\right)\right|_{\Gamma_{t}}=0 .
$$

Так как $\left.f_{1}\right|_{\Gamma_{t}}=0$, то (38) эквивалентно первому условию (16).

Нам осталось вьвести второе условие (16). Для этого рассмотрим уравнение (28.4). Проводя такие же вычисления, что и при выводе (36), (37), убеждаемся в том, что (33.4) приводит к условию Стефана (16) с правой частью

$$
f=\int_{-\infty}^{+\infty}\left(F_{\theta}^{4}-\frac{\partial}{\partial \tau} K(\chi)\left(\left\langle\nabla \psi, \nabla \theta_{1}^{ \pm}\right\rangle-\left\langle\nabla \psi_{1}, \nabla \theta^{ \pm}\right\rangle\right)\right) d \tau
$$

Теперь определим продолжение $\theta_{1}+f_{1} V_{1}+\left.g_{1}\right|_{\Gamma_{t}}$ вне поверхности $\Gamma_{t}$. Для этого обозначим через $\theta_{1}^{ \pm}=\theta_{1}^{ \pm}(x, t)$ решение задачи Стефана с граничным условием $(38)$ в $\Omega_{0}^{ \pm}$, и пусть $\widetilde{\theta}_{1}^{ \pm}(x, t)$ - гладкие продолжения $\theta_{1}^{ \pm}$в области $\Omega^{\mp}$ соответственно такие, что каждая из функций $\widetilde{\theta}_{1}^{ \pm}$удовлетворяет уравнению теплопроводности (25.1).

Тогда, полагая $f_{1} V_{1}+g_{1}=\left(\widetilde{\theta}_{1}^{+}-\widetilde{\theta}_{1}^{-}\right) \chi / 2, \theta_{1}=\left(\widetilde{\theta}_{1}^{+}+\widetilde{\theta}_{1}^{-}\right) / 2$, определяем в $\Omega$ первую поправку асимптотики $\theta$.

Таким образом, вычисление младших членов асимптотического разложения $\theta$ и $\varphi$ сводится к решению классической задачи Стефана и решению обькновенных уравнений $(27.2),(28.4)$. Построение следующих членов асимптотики проводится аналогично указанной вьше схеме.

3. Модифицированная задача Стефана. Рассмотрим теперь случай, когда параметры $a, \tau, \xi$ в (1) связаны соотношением (5). Тогда задача сводится к системе $(7),(8)$ при $\alpha=2$.

Здесь мы только сформулируем результат, введя предварительно вспомогательные функции. Пусть $\theta^{ \pm}, \psi$ - решение модифицированной задачи Стефана

$$
\begin{gathered}
\frac{\partial \theta^{ \pm}}{\partial t}=\left\langle\nabla, K^{ \pm} \nabla \theta\right\rangle, \\
{\left.\left[\theta^{ \pm}\right]\right|_{\Gamma_{t}}=0,\left.\quad\left[K^{ \pm} \frac{\partial \theta^{ \pm}}{\partial \nu}\right]\right|_{\Gamma_{t}}=2 V_{\nu},} \\
\left.\theta^{ \pm}\right|_{\Sigma}=\left.h(x, t)\right|_{\Sigma},\left.\quad \theta^{ \pm}\right|_{t=0}=\theta_{0}^{ \pm}(x), \\
\left.\varkappa_{2} \theta^{ \pm}\right|_{\Gamma_{t}}=\mathscr{K}_{t}+\varkappa_{1} V_{\nu},
\end{gathered}
$$

где $\varkappa_{2}=\varkappa / \sqrt{2}, \mathscr{K}_{t}=\operatorname{div}(\nabla \psi /|\nabla \psi|)-$ средняя кривизна $\Gamma_{t}, V_{\nu}=1 /|\nabla \psi|-$ скорость движения границы $\Gamma_{t}$ в направлении нормали. Пусть $\widetilde{\theta}^{ \pm}-$гладкие продолжения функции $\theta^{ \pm}$в $\Omega_{t}^{\mp}$ такие, что $\widetilde{\theta}^{ \pm}$удовлетворяют уравнению теплопроводности (39). 
ТЕОРема 2. Пусть при $t \leqslant T$ существует классическое решение задачи (39)-(42). Тогда при $t \leqslant T$ асимптотическое решение типа кинка системы (7), (8) при $\alpha=2$ u.меeт вид

$$
\begin{aligned}
& \varphi(x, t, \varepsilon)=\chi(\tau, x, t)+\varepsilon \frac{\partial \beta}{\partial \nu} \frac{\tau^{2}}{2} \frac{\partial \chi}{\partial \tau}(\tau, x)+O\left(\varepsilon^{2}\right), \\
& \theta(x, t, \varepsilon)=\frac{1}{2}\left(\widetilde{\theta}^{+}(x, t)+\widetilde{\theta}^{-}(x, t)\right)+\frac{1}{2}\left(\widetilde{\theta}^{+}(x, t)-\widetilde{\theta}^{-}(x, t)\right) \chi(\tau, x)+O(\varepsilon),
\end{aligned}
$$

$2 \partial e$

$$
\tau=\frac{t-\psi(x)}{\varepsilon}+\psi_{1}, \quad \chi=\operatorname{th} \frac{\tau}{\sqrt{2}|\nabla \psi|},
$$

$\psi_{1}=\psi_{1}(x)$ - некоторая гладкая функиия.

Доказательство теоремы, а также вычисление $\psi_{1}$ проводится, как в п. 2 (см. также [14], [16]). Различие состоит в том, что быстро меняющаяся переменная имеет вид

$$
\tau=\frac{t-\psi(x)}{\varepsilon}+\psi_{1}+\cdots
$$

Модельные уравнения для членов асимптотики $\varphi$ получаются такими же, как и в предыдущей задаче, т.е. вида $(26)$ и $(27 . k)$, но правые части вычисляются по другим формулам. В частности, уравнение для первой поправки имеет правую часть

$$
F_{1}^{\varphi}=\left(2\langle\nabla \psi, \nabla\rangle+\Delta \psi+\varkappa_{1}\right) \frac{\partial \chi}{\partial \tau}-\frac{1}{2} \varkappa \check{\theta}_{0}\left(1-\chi^{2}\right)+2\left\langle\nabla \psi, \nabla \psi_{1}\right\rangle \frac{\partial^{2} \chi}{\partial \tau^{2}}
$$

Таким образом, условие ортогональности

$$
\int_{-\infty}^{+\infty} F_{1}^{\varphi} \frac{\partial \chi}{\partial \tau} d \tau=0
$$

приводит не к (12), а к граничному условию Гиббса-Томсона (42).

\section{СПИСОК ЦИТИРОВАННОЙ ЛИТЕРАТУРЫ}

[1] Caginalp G. An analysis of a phase field model of a free boundary // Arch. Rational Mech. Anal. 1986. V. 92. P. 205-245.

[2] Caginalp G., Chen X. Phase field equations in the singular limit of sharp interface problem // On the Evolution of Phase Boundaries / ed. M. Gurtin, G. B. McFadden. IMA Vol. Math. Appl. V. 43. New York: Springer, 1992. P. 1-28.

[3] Modica L. The gradient theory of phase translations and the minimal interphase criterion // Arch. Rational Mech. Anal. 1986. V. 98. P. 123-142.

[4] Luckhaus S., Modica L. The Gibbs-Thomson relation within the gradient theory of phase translations // Arch. Rational Mech. Anal. 1989. V. 107. P. 71-83.

[5] Luckhaus S. Solutions of the two-phase Stefan problem with the Gibbs-Thomson law for the melting temperature // European J. Appl. Math. 1990. V. 1. P. 101-111. 
[6] Плотников П.И., Старовойтов В.Н. Задача Стефана как предел системы фазового поля // Дифференц. уравнения. 1993. Т. 29. №3. С. 461-471.

[7] Soner H.M. Convergence of the phase field equations to the Mullins-Sekerka problem with kinetic undercooling // Arch. Rational Mech. Anal. 1995. V. 131. P. 139-197.

[8] Caginalp G. Stefan and Hele-Shaw type models as asymptotic limits of the phase field equations // Phys. Rev. A. 1990. V. 39. P. 101-111.

[9] Caginalp G., Chen X. Convergence of the phase field model to its sharp interphase limits // European J. Appl. Math. 1998. V. 9. № 4. P. 417-445.

[10] Alikakos N., Bates P. On the singular limit in a phase field model of a phase translations // Ann. Inst. H. Poincaré. Phys. Théor. 1988. V. 5. P. 1-38.

[11] Carr J., Pego R. L. Metastable patterns in solutions of $u_{t}=\varepsilon^{2} u_{x x}-f(u) / /$ Comm. Pure Appl. Math. 1989. V. 42. P. 523-576.

[12] Chen X., Elliott C. M. Asymptotics for a parabolic double obstacle problem // Proc. Roy. Soc. London. Ser. A. 1994. V. 444. P. 429-445.

[13] Nochetto R., Verdi C. Convergence of double obstacle problem to the generalized geometric motion of fronts // SIAM J. Math. Anal. 1995. V. 26. № 4. P. 1514-1526.

[14] Данилов В. Г., Омельянов Г. А., Радкевич Е. В. Асимптотика решения системы фазового поля и модифицированная задача Стефана // Дифференц. уравнения. 1995. Т. 31. № 3 . C. $483-491$.

[15] Данилов В. Г., Омельянов Г. А., Радкевич Е. В. Обоснование асимптотики решения системы фазового поля и модифицированная задача Стефана // Матем. сб. 1995. Т. 186. № 12. C. $63-80$.

[16] Омельянов Г.А., Трушков В. В. Геометрическая поправка в задаче о движении свободной границы // Матем. заметки. 1998. Т. 63. №1. С. 151-153.

[17] Маслов В. П., Омельянов Г. А. Асимптотические солитонообразные решения уравнений с малой дисперсией // УМН. 1981. Т. 36. № 3. С. 63-126.

[18] Маслов В.П., Данилов В. Г., Волосов К. А. Математическое моделирование процессов тепломассопереноса. М.: Наука, 1987. 